\title{
Enfermería en la monitorización del delirium en cuidado intensivo adulto: una revisión de alcance*
}

\author{
Nursing in delirium monitoring in adult intensive care: a scope review \\ Enfermagem do monitoramento do delirium em terapia intensiva de adultos: revisão do escopo
}

Angela María Henao Castaño ${ }^{a}$

DOI: https://doi.org/10.11144/Javeriana.ie22.emdc

Universidad Nacional de Colombia, Colombia

angmhenaocas@unal.edu.co

ORCID: https://orcid.org/0000-0003-4203-0016

Recibido: 12 Agosto 2019

Angela Camila Baquero Lizarazo

Aceptado: 20 Mayo 2020

Universidad Nacional de Colombia, Colombia

Publicado: 30 Diciembre 2020

ORCID: https://orcid.org/0000-0002-2446-645X

Nathaly González Pabón

Hospital Universitario Nacional, Colombia

ORCID: https://orcid.org/0000-0002-3486-7046

Ginna Fernanda Burgos Tulcán

Universidad Nacional de Colombia, Colombia

ORCID: https://orcid.org/0000-0003-2762-5660

Linamaría Lozano

Hospital Universitario Nacional, Colombia

ORCID: https://orcid.org/0000-0002-3227-9152

\section{Resumen:}

Introducción. El delirium es una alteración cognitiva secundaria a una situación clínica aguda que puede estar presente en el paciente crítico, y la enfermería juega un rol protagónico en la prevención, monitoreo y tratamiento no farmacológico del delirium en el paciente en unidad de cuidado intensivo. Objetivo. Identificar las investigaciones actuales que describen las estrategias que utilizan las enfermeras para la monitorización del delirium en la unidad de cuidado intensivo adulto. Método. Revisión de alcance, utilizando la pregunta orientadora equé estrategias utiliza enfermería en la monitorización del delirium en la unidad de cuidado intensivo?, estudios obtenidos en las bases de datos PubMed, Biblioteca Virtual en Salud, Science Direct y Cochrane. Se realizó una revisión crítica con el instrumento CASPe, los datos se extrajeron, y se llevaron a cabo registros en tablas de gráficos elaboradas para condensar la información. Resultados. Trece estudios se consideraron relevantes y fueron analizados. Las estrategias de monitorización del delirium empleadas por enfermería son, principalmente, el juicio clínico, la observación de la agitación y, en menor medida, el empleo de instrumentos validados para la detección. Conclusión. La enfermería requiere comprender mejor el delirium, y para esto la educación es vital para mejorar su conocimiento, y aumentar la autoconfianza y competencia en la evaluación a través del uso correcto de instrumentos.

Palabras clave: Delirium, cuidados críticos, enfermería.

\section{Abstract:}

Introduction. Delirium is a cognitive alteration secondary to an acute clinical situation, which may be present in patients in critical condition. Nursing plays a key role in the prevention, monitoring, and non-pharmacological treatment of delirium in patients in intensive care units. Objective. Identify current research projects that describe the strategies used by nurses for delirium monitoring in adult intensive care units. Methods. Scope review, using the guiding question, what strategies are used by nursing professionals in the monitoring of delirium in intensive care units? Studies were gathered from the PubMed, Virtual Health Library (Biblioteca Virtual en Salud), Science Direct, and Cochrane databases. A critical review was performed using the CASPe instrument. Data were extracted and entries were made in graphic tables created to condense the information. Results. Thirteen studies were considered

Notas de autor

a Autora de correspondencia. Correo electrónico: angmhenaocas@unal.edu.co 
relevant and were analyzed. The delirium monitoring strategies mainly used by nursing professionals were clinical judgement, observation of agitation and, to a lesser extent, the use of valid detection instruments. Conclusion. It is required for nursing professionals to gain a better understanding of delirium and to this end education is vital to improve knowledge and increase selfconfidence and competence in the assessment through the correct use of instruments.

Keywords: Delirium, critical care, nursing.

\section{Resumo:}

Introdução. O delirium é uma alteração cognitiva secundária a uma situação clínica aguda que pode estar presente em pacientes críticos, e a enfermagem tem um papel principal na prevenção, monitoramento e tratamento não farmacológico do delirium em pacientes na unidade de cuidado intensivo. Objetivo. Identificar as pesquisas atuais descrevendo as estratégias utilizadas pelos enfermeiros para o monitoramento do delirium na unidade de terapia intensiva de adultos. Método. Revisão do escopo, utilizando a questão norteadora quais as estratégias da enfermagem no monitoramento do delirium na unidade de terapia intensiva? Estudos obtidos nas bases de dados PubMed, Biblioteca Virtual em Saúde, Science Direct e Cochrane. Revisão crítica com o instrumento CASPe foi realizada, os dados extraíram-se e foram feitos registros em tabelas de gráficos elaboradas para condensar as informações. Resultados. Treze estudos consideraram-se relevantes e foram analisados. As estratégias de monitoramento do delirium usadas pela enfermagem são, principalmente, o julgamento clínico, a observação da agitação e, em menor medida, o uso de instrumentos validados para a detecção. Conclusão. A enfermagem requer melhor entendimento do delirium, e para isso a educação é vital para aprimorar seu conhecimento, e aumentar a autoconfiança e competência na avaliação através do uso certo de instrumentos.

Palavras-chave: Delirium, cuidados críticos, enfermagem.

\section{Introducción}

El delirium es un episodio agudo de insuficiencia cerebral, caracterizado por la confusión con un estado mental fluctuante, falta de atención, un nivel de conciencia alterado, pensamiento desorganizado y posibles alucinaciones; puede ocurrir en la unidad de cuidado intensivo (UCI) secundario a un evento médico agudo, relacionado con medicamentos o enfermedad (1). Puede afectar hasta el $80 \%$ de los pacientes en la UCI, y se ha asociado con mayores tasas de intubación y de mortalidad, mayor estancia, reingresos hospitalarios, riesgo de demencia e institucionalización (2). A largo plazo, genera deterioro funcional, mayor tasa de mortalidad y deterioro neurocognitivo (3).

A pesar de su importancia clínica, a menudo se diagnostica erróneamente o no es reconocido por el personal de salud que está a cargo del cuidado $(4,5)$. En los pacientes de la UCI, la falta de una evaluación cognitiva regular, la dificultad para establecer comunicación con los pacientes que reciben ventilación mecánica, la presentación frecuente de delirium hipoactivo, y la falta de adopción en las UCI de una herramienta de evaluación validada para formalizar su monitorización - a pesar de la existencia y sugerencia del uso de instrumentos como la CAM-ICU (Confusion Assessment Method for the ICU), el ICDSC (Intensive Care Delirium Screening Checklist. y la Nursing Delirium - afectan la tasa de reconocimiento $(6,7)$.

El delirium es reversible, por lo que se puede prevenir y tratar, y la detección temprana mediante un examen exhaustivo es un factor clave para la prevención de sus consecuencias (8). El enfermero, quien realiza la valoración exhaustiva en la UCI, puede ser quien identifique las fluctuaciones en el estado cognitivo de manera temprana $(9,10)$.

El reconocimiento temprano del riesgo o presencia de delirium a través de la monitorización sistemática y continua, como lo recomienda el paquete $\mathrm{ABCDEF}$ en la sigla D, Delirium Monitoring/Management, con escalas validadas como la CAM-ICU y el ICDSC, permite prevenirlo y tratarlo oportunamente (11); y esto se ha asociado con reducción en la mortalidad y estancia en la UCI (12). De esta manera, es un tema importante que debe abordarse desde enfermería, porque juega un rol protagónico en la prevención, monitoreo y tratamiento no farmacológico del delirium en el paciente en unidad de cuidado intensivo.

Se realizó una revisión de alcance a través de la pregunta ¿qué estrategias utiliza enfermería para la monitorización del delirium en la UCI?, para identificar las estrategias con el fin de orientar la implementación de estas, mejorar la detección del delirium y disminuir los resultados negativos asociados, 
pues la literatura reporta que existe una brecha de conocimiento de los enfermeros al realizar una evaluación específica del delirium en la UCI (9).

\section{Objetivo}

Identificar las investigaciones actuales a través de una revisión de alcance de literatura, que describa las estrategias que utilizan las enfermeras para la monitorización del delirium en la unidad de cuidado intensivo adulto.

\section{Método}

Se llevó a cabo una revisión de alcance, teniendo como marco de referencia los seis pasos definidos por Arskey y O’Malley citado en Peters et al. (13), que son: 1) identificación de la pregunta de investigación, 2) identificación de estudios relevantes, 3) selección de estudios, 4) graficar datos, 5) cotejar, resumir y reportar resultados, y 6) consulta. Para esta revisión se realizaron los primeros cinco pasos, dado que el último es opcional. Se han realizado revisiones similares en las que se describen las estrategias para la monitorización del delirium recomendadas por la Sociedad de Medicina de Cuidados Críticos dentro de las pautas para el Manejo del dolor, agitación y delirio (PAD), principalmente la CAM-ICU y el ICDSC (14); o se han centrado en la descripción de la estrategia $\mathrm{ABCDEF}$ como una herramienta para la prevención y tratamiento (11). Sin embargo, no se ha realizado una revisión de alcance por parte de enfermería, donde se den a conocer las estrategias de monitorización en la UCI, sino que se han centrado principalmente en los factores que contribuyen al bajo reconocimiento del delirium (3).

\section{Proceso de búsqueda y criterios de inclusión}

Para la búsqueda se utilizaron palabras claves como "delirium", "cuidado intensivo", "cuidado agudo", "enfermería", "adultos", "instrumentos" y "estrategias". Las bases de datos consultadas fueron PubMed, Biblioteca Virtual en Salud (BVS), Science Direct y Cochrane, durante los meses de mayo y junio de 2019. Los criterios de inclusión fueron estudios originales de metodología cuantitativa, cualitativa y revisiones realizadas en los últimos seis años (2014-2019), que describen estrategias para la monitorización del delirium, desarrolladas por o para enfermería en la UCI o en otros servicios que describen estrategias empleadas en la misma, con texto completo disponible, idioma español e inglés y población mayor de 18 años. Los criterios de exclusión fueron artículos en los que solo se pudo consultar el resumen, e investigaciones que no se referían a estrategias de monitorización del delirium en la UCI.

\section{Proceso de selección y extracción}

Se seleccionaron 233 estudios que cumplían con los criterios de inclusión al realizar la búsqueda. Al leer el título y el resumen de manera independiente, los investigadores se seleccionaron 33 artículos para su revisión en texto completo. Posteriormente, se realizó crítica con el instrumento CASPe de manera independiente por los investigadores. Al finalizar este proceso y el consenso entre investigadores, se seleccionaron 13 artículos para ser incluidos en la revisión del alcance, los cuales se presentan por medio del diagrama Prisma (15) (este proceso está descrito y detallado en la Figura 1). Los factores determinantes evaluados por cada investigador para la selección de los estudios fueron el objetivo, la intervención o instrumentos empleados o descritos, los 
resultados, la discusión y las conclusiones. Este estudio cumplió con los criterios éticos relacionados con la propiedad intelectual.

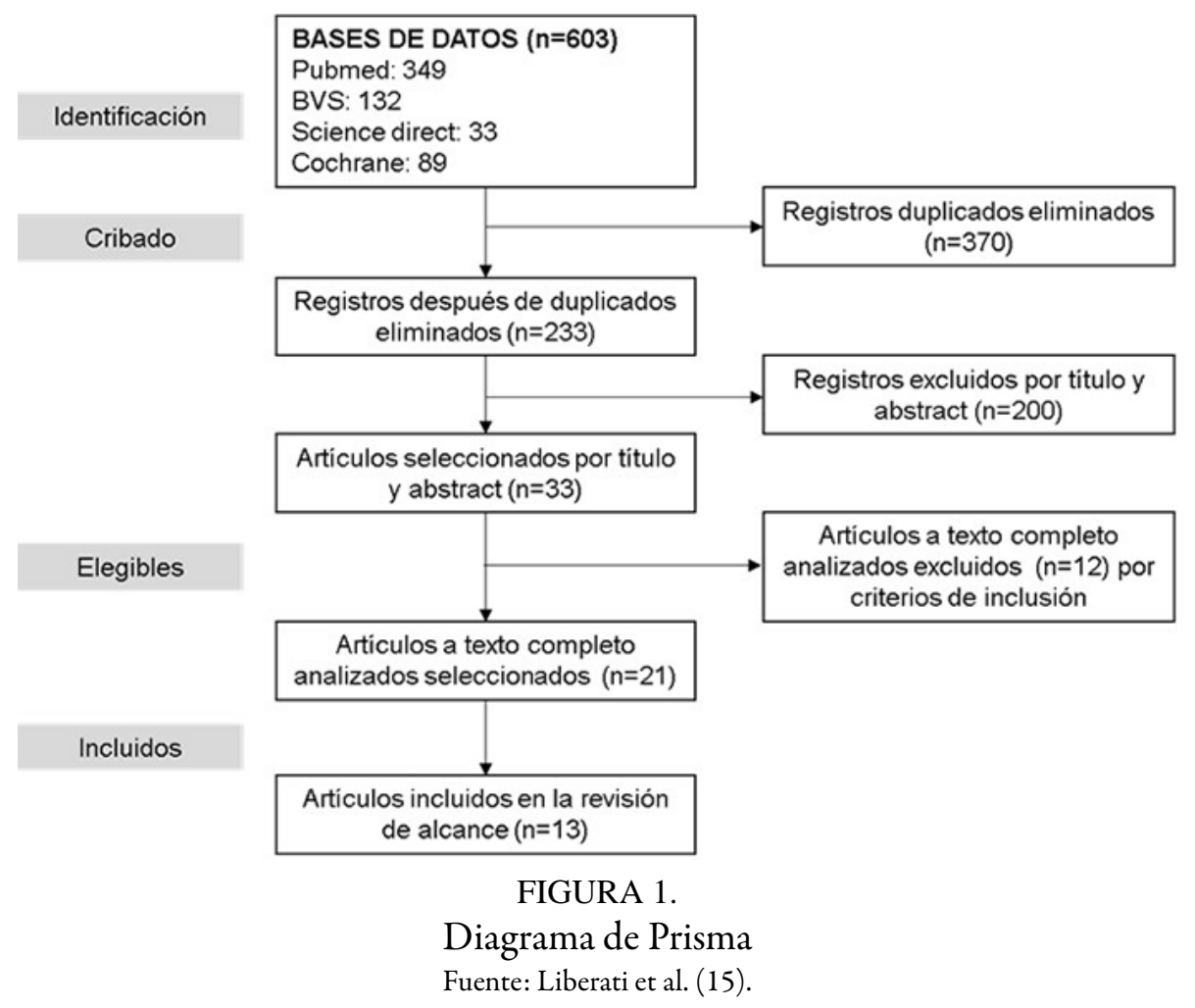

\section{Resultados}

En esta revisión de alcance se incluyeron trece estudios. El promedio de artículos seleccionados en las revisiones fue 59, el promedio de tamaño de muestra de los artículos de cohorte fue de 645 pacientes, y el promedio de la muestra del estudio de encuesta y el correlacional fue de 405 pacientes. El estudio correlacional relacionó el sistema de prestación de servicios con la evaluación del delirium, resaltando que el personal de enfermería que utilizaba el sistema centrado en el paciente evalúa el delirium con mayor frecuencia (16) (ver Tabla 1). 
TABLA 1.

Artículos incluidos en la revisión de alcance

\begin{tabular}{|c|c|c|c|}
\hline Estudio/año & Idioma/pais & Diseño & $\begin{array}{l}\text { Monitorización del delirium por } \\
\text { enfermeria }\end{array}$ \\
\hline $\begin{array}{l}\text { Lamond et } \\
\text { al., } 2018 \\
\text { (16) }\end{array}$ & $\begin{array}{l}\text { Inglés, } \\
\text { Reino } \\
\text { Unido }\end{array}$ & $\begin{array}{l}\text { Revisión } \\
\text { narrativa }\end{array}$ & $\begin{array}{l}\text { Enfermeras deben asegurarse de utilizar } \\
\text { herramientas actualizadas y relevantes } \\
\text { para diagnosticar el delirium, como la } \\
\text { CAM-ICU o el ICDSC. }\end{array}$ \\
\hline $\begin{array}{l}\text { Özsaban y } \\
\text { Acaroglu, } \\
2015(17)\end{array}$ & $\begin{array}{l}\text { Inglés, } \\
\text { Turquia }\end{array}$ & $\begin{array}{c}\text { Estudio } \\
\text { descriptivo y } \\
\text { correlacional }\end{array}$ & $\begin{array}{l}\text { El empleo de un sistema de prestación de } \\
\text { servicios centrado en la paciente mejora } \\
\text { la relación enfermera-paciente y permite } \\
\text { un seguimiento continuo y rápido. }\end{array}$ \\
\hline $\begin{array}{l}\text { Nydahl et } \\
\text { al., } 2018 \\
\text { (18) }\end{array}$ & $\begin{array}{c}\text { Inglés, } \\
\text { Alemania }\end{array}$ & $\begin{array}{l}\text { Estudio de } \\
\text { encuesta }\end{array}$ & $\begin{array}{l}\text { El aumento de la implementación de los } \\
\text { objetivos diarios interprofesionales en el } \\
\text { contexto del delirium mejora la } \\
\text { evaluación y tratamiento del mismo. }\end{array}$ \\
\hline $\begin{array}{c}\text { Middle y } \\
\text { Miklancie, } \\
2015(19)\end{array}$ & $\begin{array}{l}\text { Inglés, } \\
\text { Estados } \\
\text { unidos }\end{array}$ & $\begin{array}{l}\text { Revisión de } \\
\text { literatura }\end{array}$ & $\begin{array}{l}\text { El uso de herramientas estandarizadas de } \\
\text { evaluación del delirium puede ayudar a } \\
\text { las enfermeras a reconocerlo y evaluarlo } \\
\text { en sus pacientes. }\end{array}$ \\
\hline $\begin{array}{l}\text { Dale et al., } \\
2014(20)\end{array}$ & $\begin{array}{l}\text { Inglés, } \\
\text { Estados } \\
\text { Unidos }\end{array}$ & $\begin{array}{c}\text { Estudio de } \\
\text { cohorte }\end{array}$ & $\begin{array}{l}\text { La implementación de un protocolo de } \\
\text { sedación, analgesia y delirium } \\
\text { actualizado, realizado de forma } \\
\text { interdisciplinar, se asocia con una mayor } \\
\text { evaluación de los niveles de sedación y } \\
\text { reducción del delirium. }\end{array}$ \\
\hline $\begin{array}{c}\text { Zoremba y } \\
\text { Coburm, } \\
2019(21)\end{array}$ & $\begin{array}{l}\text { Inglés, } \\
\text { Alemania }\end{array}$ & $\begin{array}{l}\text { Revisión de } \\
\text { literatura }\end{array}$ & $\begin{array}{l}\text { El diagnóstico de delirium debe estar } \\
\text { estrechamente relacionado con el dolor y } \\
\text { la agitación, y el empleo de estrategias } \\
\text { multidimensionales a través del trabajo } \\
\text { multidisciplinar permite reducir la } \\
\text { incidencia de mismo. }\end{array}$ \\
\hline $\begin{array}{l}\text { Boettger et } \\
\text { al., 2018 } \\
\text { (22) }\end{array}$ & $\begin{array}{l}\text { Inglés, } \\
\text { Suiza }\end{array}$ & $\begin{array}{c}\text { Estudio } \\
\text { descriptivo de } \\
\text { cohorte }\end{array}$ & $\begin{array}{l}\text { El ICDSC en un umbral de }>3 \text { mejora su } \\
\text { sensibilidad y se convierte en un } \\
\text { instrumento de detección que enfermeria } \\
\text { puede utilizar para identificar de manera } \\
\text { temprana el delirium. }\end{array}$ \\
\hline $\begin{array}{l}\text { Gélinas et } \\
\text { al., } 2018 \\
\text { (23) }\end{array}$ & $\begin{array}{l}\text { Inglés, } \\
\text { Estados } \\
\text { Unidos }\end{array}$ & $\begin{array}{c}\text { Revisión } \\
\text { sistemática }\end{array}$ & $\begin{array}{l}\text { El conocimiento de las propiedades } \\
\text { psicométricas de las herramientas de } \\
\text { detección del delirium permite a las } \\
\text { enfermeras de cuidados criticos elegir } \\
\text { una herramienta de evaluación del } \\
\text { mismo. }\end{array}$ \\
\hline $\begin{array}{l}\text { Van } \\
\text { Velthuijsen } \\
\text { et al., } 2016 \\
\text { (24) }\end{array}$ & $\begin{array}{l}\text { Inglés, } \\
\text { Paises } \\
\text { Bajos }\end{array}$ & $\begin{array}{c}\text { Revisión } \\
\text { sistemática }\end{array}$ & $\begin{array}{l}\text { La escala Nu-DESC puede ser el } \\
\text { instrumento más apropiado para su uso } \\
\text { diario en la detección del delirium por } \\
\text { enfermmeria, pues su aplicación es rápida y } \\
\text { está basada en la observación. }\end{array}$ \\
\hline $\begin{array}{l}\text { Jones et al., } \\
2018(25)\end{array}$ & $\begin{array}{l}\text { Inglés, } \\
\text { Estados } \\
\text { Unidos }\end{array}$ & $\begin{array}{c}\text { Revisión } \\
\text { sistemática }\end{array}$ & $\begin{array}{l}\text { La gravedad del delirium es un aspecto } \\
\text { que puede ser evaluado por profesionales } \\
\text { de la salud a través de instrumentos de } \\
\text { alta calidad como el método de } \\
\text { evaluación de la confusión puntaje de } \\
\text { severidad, Examen de Estado } \\
\text { Confusional, Delirium-o-Meter, Escala } \\
\text { de Observación del Delirium y Escala de } \\
\text { Evaluación de Delirium. }\end{array}$ \\
\hline $\begin{array}{c}\text { Boettger et } \\
\text { al., 2017 } \\
\text { (26)e }\end{array}$ & $\begin{array}{l}\text { Inglés, } \\
\text { Reino } \\
\text { Unido }\end{array}$ & $\begin{array}{c}\text { Estudio } \\
\text { descriptivo y de } \\
\text { cohorte }\end{array}$ & $\begin{array}{l}\text { La determinación del subtipo motor del } \\
\text { delirium es importante para la detección } \\
\text { y tratamiento. Enfermeria puede } \\
\text { valorarlo empleando instrumentos } \\
\text { validados y confiables como el DMSS-4. }\end{array}$ \\
\hline $\begin{array}{c}\text { Khan et al., } \\
2017 \text { (27) }\end{array}$ & $\begin{array}{l}\text { Inglés, } \\
\text { Estados } \\
\text { Unidos }\end{array}$ & $\begin{array}{c}\text { Estudio de } \\
\text { cohorte } \\
\text { observacional }\end{array}$ & $\begin{array}{l}\text { El empleo de una herramienta para } \\
\text { evaluar la gravedad del delirium, comola } \\
\text { CAM-ICU-7, ayuda a evitar que se pasen } \\
\text { por alto los pacientes de menor gravedad, } \\
\text { a los que se les pueden iniciar } \\
\text { intervenciones multidisciplinarias } \\
\text { tempranas. }\end{array}$ \\
\hline $\begin{array}{l}\text { Rowley- } \\
\text { Conwy, } \\
2018(28)\end{array}$ & $\begin{array}{l}\text { Inglés, } \\
\text { Reino } \\
\text { Unido }\end{array}$ & $\begin{array}{l}\text { Revisión de } \\
\text { literatura }\end{array}$ & $\begin{array}{l}\text { Las principales estrategias que utilizan } \\
\text { las enfermeras para la monitorización del } \\
\text { delirium son escalas como el CAM-ICU } \\
\text { y el ICDSC, la observación de la } \\
\text { agitación y la evaluación de la capacidad } \\
\text { del paciente de seguir órdenes. }\end{array}$ \\
\hline
\end{tabular}

En general, las estrategias que emplean las enfermeras para la monitorización del delirium son la observación de la agitación, la evaluación de las funciones cognitivas del paciente y la aplicación de instrumentos de detección. Los estudios sugieren la utilización, principalmente, de instrumentos validados como el CAM-ICU, la Nu-DESC y el $\operatorname{ICDSC}(16,17,18,22,23,24)$, la aplicación de estrategias 
multidisciplinarias $(18,21)$ y de protocolos de sedación, analgesia y delirium (20). Los resultados se exponen en seis categorías relevantes para responder la pregunta central planteada.

\section{Prácticas y percepciones en la evaluación del delirium en la UCI}

Özsaban y Acaroglu (17) realizaron un estudio descriptivo correlacional para determinar las prácticas y percepciones de las enfermeras respeto a la evaluación del delirium en la UCI. Se determinó que el 67.8\% realizaron evaluaciones rutinarias del delirium, $89.3 \%$ solicitaron consultas de psiquiatría para el diagnóstico, y solo utilizan herramientas de evaluación el 14.7\% de las enfermeras. De las herramientas para la detección, las más utilizadas fueron la Nu-DESC (6.3\%), CAM-ICU (4.4\%) y el ICDSC (4\%). Casi la mitad de las enfermeras (49.2\%) monitorean los síntomas del delirium una vez cada 24 horas, y el $37.9 \%$ lo evaluaron cada hora. Los síntomas identificados con mayor frecuencia fueron el aumento o disminución de la actividad psicomotora y la desorientación. El 52.2\% de las enfermeras consideran el delirium como un problema grave, y solo el 12.1\% utilizó el modelo de atención centrado en el paciente, lo que se asocia con una evaluación temprana del delirium.

Nydahl et al. (18) evaluaron el manejo del delirium en enfermeras y médicos que trabajan en UC, y encontraron que las enfermeras, (60.5\%) más que los médicos (48.5\%), informaron sobre la detección del delirium cuando este se sospechaba, y más del $50 \%$ utiliza instrumentos validados para evaluar a los pacientes.

\section{Educación de enfermería en la monitorización del delirium}

Middle et al. (19) encontraron que se podría mejorar el conocimiento e implementación de las herramientas para evaluar y detectar el delirium por parte de los enfermeros de cuidados agudos, utilizando estrategias útiles como de programas multifacéticos, el empleo de las herramientas en los entornos apropiados, estudios de caso, la simulación, la realización de rondas en cuidados intensivos, investigación de la perspectiva del paciente y la exigencia de una certificación en delirium similar a la exigida en reanimación cardiopulmonar.

\section{Cuidado de enfermería}

Enfermería participa de forma interdisciplinaria en el cuidado del paciente crítico. Las guías de analgesia, sedación y delirium son un ejemplo de una estrategia en la que enfermería debe ejercer su rol de cuidado teniendo en cuenta las recomendaciones. Dale et al. (20) evaluaron la asociación de un protocolo de analgesia, sedación y delirium con la mejora del proceso y calidad de los resultados del paciente en una UCI de trauma. El protocolo se caracterizó por promover la reducción de la sedación, proporcionar sedación después de aliviar y tratar el dolor, evaluar regularmente el delirium para evitar las benzodiazepinas, y documentar la puntuación del RASS cada 4 horas y de la CAM-ICU dos veces al día. La implementación se asoció con una mayor frecuencia en la evaluación de la sedación, una puntuación RASS más alta (sedación más ligera), disminución de la dosis media de benzodiazepinas, una reducción significativa de la carga del delirium y disminución de la duración media de la ventilación mecánica, la estancia en la UCI y la estancia hospitalaria. Según Zoremba y Coburn (21), la guía sobre analgesia, sedación y manejo del delirium en cuidados intensivos recomienda realizar un examen de delirium al menos cada 8 horas mediante un instrumento de detección validado. 


\section{Evaluación del delirium}

La evaluación del delirium requiere que las enfermeras puedan hacerla con precisión, por tanto, la práctica recomendada es la evaluación mediante observación diaria para detectar cambios en el comportamiento. Sin embargo, se describe que la precisión del diagnóstico de delirium sin una herramienta es deficiente (sensibilidad: 29\%), por lo que las enfermeras deben asegurarse de utilizar herramientas de detección actualizadas y relevantes para el diagnóstico (16). La CAM-ICU es la herramienta más adecuada $(16,24)$.

Jones et al. (25) realizó una revisión sistemática de los instrumentos que evalúan la gravedad del delirium, argumentando que estos permiten realizar un seguimiento y monitoreo durante el curso clínico y la recuperación del delirium, y dan información pronóstica que respalda y ayuda a evaluar las necesidades del paciente y la carga de atención. Seis instrumentos fueron considerados ideales para evaluar la gravedad del delirium, estos fueron el Método de Evaluación de la Confusión-Puntaje de Severidad (CAM-S), Examen de Estado Confusional (CSE), Delirium-O-Meter (DOM), Escala de Observación de Delirium (DOS), Escala de Evaluación del Delirium (DRS) y Escala de Evaluación de Delirium Memorial (MDAS).

Gélinas et al. (23) evaluaron las propiedades psicométricas de las herramientas de evaluación del delirium para adultos en estado crítico, donde las puntuaciones totales ponderadas oscilaron entre 0 y 20 . Los resultados arrojaron puntuaciones ponderadas de la CAM-ICU (19.6) y ICDSC (19.2), indicando muy buenas propiedades psicométricas. Las propiedades psicométricas del Nu-DESC fueron moderadas (13.6), las del DDS fueron bajas (11.2), y las del CTD (Cognitive Test for Delirium) fueron muy bajas (8.2).

\section{Barreras para la monitorización (evaluación) del delirium}

Özsaban y Acaroglu (17) describen como barreras para la evaluación del delirium por parte de las enfermeras, los pacientes bajo sedación (73.8\%) o ventilados (66.1\%), la complejidad de las herramientas de diagnóstico (65.8\%) y el tiempo requerido para la observación. Nydahl et al. (18) reportaron como barreras la falta de tiempo, seguida por la falta de conocimiento sobre el delirium, así como las brechas de comunicación entre las profesiones. Según Rowley-Conwy (28), existen otras barreras como la pobre colaboración médicoenfermera y la falta de apoyo del personal superior, de tiempo, de conocimiento del delirium y de confianza con la herramienta de evaluación y en la realización de la evaluación. Lamond et al. (16) describen que la intubación es una barrera que afecta el uso de herramientas de detección, sin embargo, declara que la CAMICU puede ser usada en pacientes intubados, y la ICDSC en paciente intubados y que reciben sedación. Por tanto, enfermería debe seleccionar la herramienta adecuada en función del estado del paciente.

\section{Estrategias de prevención del delirium}

Zoremba y Coburn (21), en su revisión de literatura, presentan algunas de las estrategias multidimensionales y multidisciplinarias de prevención del delirium, que en su implementación se asocian a la reducción de la incidencia del mismo. Estas incluyen movilización temprana, reorientación, ingesta nutricional, mejora del sueño, manejo adecuado del dolor y evitar la polifarmacia. La ausencia de dolor es un componente esencial, las medidas de estimulación deben realizarse durante el día y las medidas de promoción del sueño durante la noche, además se debe evitar la sedación excesiva (RASS < -1). La terapia física y ocupacional demostró reducción en la tasa de delirium de $41 \%$ a $28 \%$. Los altos niveles de ruido en la UCI pueden causar estrés e insomnio, pudiendo desencadenar el delirium, por lo que es necesario tomar medidas para mejorar la calidad del sueño (21). 


\section{Discusión}

Esta revisión brinda un análisis de la monitorización del delirium por enfermería en la UCI, evidenciando que existen diversos instrumentos y estrategias para evaluarlo, pero que su aplicación, así como la evidencia científica en la práctica clínica, es poca. El enfermero juega un rol importante en la monitorización del delirium en la UCI, lo que se ha enfatizado en diferentes estudios como el de Faught (29), donde la enfermera se encuentra en una posición estratégica para prevenirlo. Pero a pesar de esto, el entorno de la UCI presenta barreras que hacen que se preste poca atención a este tipo de episodios y su diagnóstico pase por alto; lo que también se ha afirmado en estudios como el de Faría y Moreno (30), donde hasta el 66\% de los casos de delirium no se diagnostican, y el de Arumugam et al. (31), donde el diagnóstico se dificulta si no se emplean herramientas de detección.

Este estudio reveló que más del $60 \%$ de los enfermeros evalúan de forma rutinaria el delirium, aunque el empleo de instrumentos validados en la práctica es poco. Esto no coindice con lo descrito por Selim y Ely (32) y Xing et al. (33), donde solo el 26.8\% y el 25.62\% de los profesionales de la UCI, respectivamente, evalúan el delirium de manera rutinaria $(32,33)$. Sin embargo, sí se reconoce por Tostes et al. (34), que los profesionales de la salud no utilizaron herramientas estandarizadas, y se basaron en su juicio clínico y experiencia.

Las herramientas más utilizadas para la detección por enfermería son la Nu-DESC, el CAM-ICU y el ICDSC, esto corresponde a lo reportado en otros estudios, donde se afirma que los profesionales emplean la CAM-ICU y el ICDSC como principales herramientas de detección $(33,35)$, aunque también emplean otras estrategias como observación de la agitación y la consulta psiquiátrica (35).

La detección y la evaluación del delirium se puede mejorar a través de la educación a las enfermeras, lo que se evidencia con hallazgos de otros estudios donde consideran que, para reconocer el delirium, se necesita una mejor comprensión y preparación antes de poder realizar una valoración rutinaria, y que la educación es vital $(36,37)$. Además, para ser efectiva debe incorporar métodos didácticos y pedagógicos, utilizar estrategias tanto en vivo como basadas en la web, ser permanente y reforzar el cumplimiento en la detección (32, 37, 38). Gesin et al. (38), Lieow et al. (39), Van Velthuijsen et al. (40), Norman y Taha (41) y Ramoo et al. (42) comprueban que la educación resultó en mayor conocimiento, autoconfianza y competencia de las enfermeras en la evaluación del delirium, mejor actitud, mayor proporción de pacientes evaluados y empleo de instrumentos de manera correcta.

La aplicación de protocolos de agitación, sedación y delirium se asocia con mayor evaluación de la sedación, puntajes del RASS más altos, menos carga de delirium y menos duración de la ventilación mecánica, estancia en la UCI y hospitalización. Mansouri et al. (43) también reportaron reducción de la duración de la ventilación mecánica, de la estancia en la UCI y de la tasa de mortalidad cuando se aplicó el protocolo.

El uso de herramientas de detección del delirium por parte de enfermería es imperativo porque la precisión de su diagnóstico sin una herramienta es deficiente, lo que se evidencia en la literatura, donde las enfermeras, sin el empleo de una herramienta, tuvieron un porcentaje de reconocimiento del delirium de $27 \%$ y $34.8 \%$ $(44,45)$.

Xing et al. (33), Devlin et al. (45), Grover (47), Ramoo et al. (42), Andrews et al. (48), Elliot (49), y Powell et al. (50) reconocen como barreras la falta de herramientas de detección adecuadas o la complejidad en su empleo, la gran carga de trabajo, la falta de conocimiento del delirium, la falta de confianza en la realización de la evaluación, la dificultad de uso en pacientes intubados y con ventilación mecánica, y la falta de respuesta a los hallazgos por el personal médico, lo que se asemeja a las barreras reportadas en esta revisión.

Se identifica la necesidad de aplicar estrategias multidimensionales que permitan la prevención y reducción de la incidencia del delirium, y que incluyan medidas para favorecer la movilización temprana, la reorientación, la nutrición, el sueño y el manejo adecuado del dolor. Este tipo de estrategia es similar al paquete ABCDEF y a las guías para la prevención y el tratamiento del dolor, sedación y el delirium, que combinan múltiples estrategias de práctica basadas en evidencia con la rutina diaria para mejorar los resultados generales 
para los pacientes y permitir una reducción sistemática en los factores de riesgo modificables para el delirium $(51,52,53,54)$.

Las limitaciones de este estudio incluyen la posibilidad de haber omitido estudios relevantes por la exclusión de artículos publicados en idiomas diferentes al inglés y español, y la búsqueda en cuatro bases de datos (PubMed, Biblioteca Virtual en Salud, Science Direct y Cochrane). El escaso tiempo de búsqueda fue otra limitación, pues se realizó entre mayo y junio de 2019. Además, la falta de evaluación crítica de los estudios seleccionados, propio de un scopingreview, puede ocasionar que los resultados no sean utilizados en la práctica.

\section{Conclusión}

La enfermera ejerce un rol esencial en la identificación del delirium en los pacientes de cuidados intensivos, por lo que es un requisito fundamental el saber evaluar esta alteración, sin embargo, hay dificultades para el reconocimiento del delirium por el modelo de atención de enfermería, barreras como la falta de conocimiento o la falta de confianza en la realización de la evaluación, lo que dificulta una adecuada monitorización, donde la educación es primordial para superar estas barreras. Las estrategias de monitorización empleadas por enfermería son principalmente el juicio clínico y la observación de la agitación, además las herramientas de detección, en menor medida. Las más utilizadas por las enfermeras en la unidad de cuidados intensivos son $\mathrm{Nu}$-DESC, CAM-ICU y el ICDSC, las cuales han reportado buenas propiedades psicométricas.

\section{Referencias}

1. Page V, Wesley E. What is delirium in critical care? Critic Care. 2008;12(supl. 3):1-13.

2.Zamoscik K, Godbold R, Freeman P. Intensive care nurses' experiences and perceptions of delirium and delirium care. Intensive Crit Care Nurs [Internet]. 2017;40(jun.):94-100. https://www.doi.org/10.1016/j.iccn.2017.01.003

3. El Hussein M, Hirst S, Salyers V. Factors that contribute to underrecognition of delirium by registered nurses in acute care settings: a scoping review of the literature to explain this phenomenon. J Clin Nurs [Internet]. 2015;24(abr.):906-15. https://www.doi.org/10.1111/jocn.12693

4. Martins S, Fernandes L. Delirium in Elderly People: A Review. Front Neurol [Internet]. 2012 3(jun.). https://ww w.doi.org/10.3389/fneur.2012.00101

5. Panitchote A, Tangvoraphonkchai K, Suebsoh N, Eamma W, Chanthonglarng B, Tiamkao S, et al. Underrecognition of delirium in older adults by nurses in the intensive care unit setting. Aging Clin Exp Res [Internet]. 2015 feb. 12.;27(5):735-740. https://www.doi.org/10.1007/s40520-015-0323-6

6. Devlin J, Verceles A, Morris P, Ely E, Balas M. Adapting the ABCDEF Bundle to Meet the Needs of Patients Requiring Prolonged Mechanical Ventilation in the Long-Term Acute Care Hospital Setting: Historical Perspectives and Practical Implications. Semin Respir Crit Care Med [Internet]. 2016;37(1):119-135. https:// www.doi.org/10.1055/s-0035-1570361

7. Öztürk Birge A, Tel Aydin H. The effect of nonpharmacological training on delirium identification and intervention strategies of intensive care nurses. Intensive Crit Care Nurs [Internet]. 2017;41(ag.):33-42. https://www.doi.o $\mathrm{rg} / 10.1016 /$ j.iccn.2016.08.009

8. Jeong E, Chang SO. Exploring nurses' recognition of delirium in the elderly by using Q-methodology. Jpn J Nurs Sci [Internet]. 2018;15(4):298-308. https://www.doi.org/10.1111/jjns.12199

9. Smith JM, Van Aman MN, Schneiderhahn ME, Edelman R, Ercole PM. Assessment of Delirium in Intensive Care Unit Patients: Educational Strategies. J Contin Educ Nurs [Internet]. 2017;48(5):239-244. https://www.doi.o rg/10.3928/00220124-20170418-09 
10. Hargrave A, Bastiaens J, Bourgeois JA, Neuhaus J, Josephson SA, Chinn J, et al. Validation of a Nurse-Based Delirium-Screening Tool for Hospitalized Patients. Psychosomatics [Internet]. 2017;58(6):594-603. https://w ww.doi.org/10.1016/j.psym.2017.05.005

11. Marra A, Ely EW, Pandharipande PP, Patel MB. The ABCDEF Bundle in Critical Care. Crit Care Clin [Internet]. 2017;33(2):225-243. https://www.doi.org/10.1016/j.ccc.2016.12.005

12. Luetz A, Weiss B, Boettcher S, Burmeister J, Wernecke KD, Spies C. Routine delirium monitoring is independently associated with a reduction of hospital mortality in critically ill surgical patients: A prospective, observational cohort study. J Crit Care [Internet]. 2016;35(oct.):168-73. https://www.doi.org/10.1016/j.jcrc.2016.05.028

13. Peters MDJ, Godfrey CM, Mclnerney P, Baldini C, Khalil H, Parker D. The Joanna Briggs Institute. Reviewers' Manual 2015. Methodology for JBI Scoping Reviews. Adelaida (Australia): The Joanna Briggs Institute; 2015.

14. Kotfis K, Marra A, Ely EW. ICU delirium, a diagnostic and therapeutic challenge in the intensive care unit. Anaesthesiol Intensive Ther [Internet]. 2018;50(2):160-167. https://www.doi.org/10.5603/AIT.a2018.0011.

15. Liberati A, Altman DG, Tetzlaff J, Mulrow C, Gøtzsche PC, Ioannidis JPA, et al. La declaración Prisma para informar revisiones sistemáticas y metaanálisis de estudios que evalúan las intervenciones de atención médica: explicación y elaboración. PLoS Medicine [Internet]. 2009;6(7): 1-28. https://www.doi.org/10.1371/journal. pmed. 1000100

16. Lamond E, Murray S, Gibson C. Delirium screening in intensive care: A life saving opportunity. Intensive Crit Care Nurs [Internet]. 2018;44(feb.):105-109. https://www.doi.org/10.1016/j.iccn.2017.04.014

17. Özsaban A, Acaroglu R. Delirium assessment in intensive care units: practices and perceptions of Turkish nurses. Nurs. Crit. Care [Internet]. 2016 Sep;21(5):271-278. https://doi.org/10.1111/nicc.12127

18. Nydahl P, Dewes M, Dubb R, Hermes C, Kaltwasser A, Krotsetis S, et al. Survey among critical care nurses and physicians about delirium management. Nurs. Crit. Care [Internet]. 2018;23(1):23-29. https://www.doi.org/1 $0.1111 /$ nicc. 12299

19. Middle B, Miklancie M. Strategies to improve nurse knowledge of delirium: a call to the adult-gerontology clinical nurse specialist. Clin Nurse Spec [Internet]. 2015;29(4):218-229. https://www.doi.org/10.1097/NUR.00000 00000000138

20. Dale C, Kannas D, Fan V, Daniel S, Deem S, Yanez N, et al. Improved Analgesia, Sedation, and Delirium Protocol Associated with Decreased Duration of Delirium and Mechanical Ventilation. Ann. Am. Thorac. Soc [Internet]. 2014;11(3):367-374. https://www.doi.org/10.1513/AnnalsATS.201306-210OC

21. Zoremba N, Coburn M. Acute confusional states in hospital. Dtsch Arztebl Int [Internet]. 2019;116(7):101-106. https://www.doi.org/10.3238/arztebl.2019.0101

22. Boettger S, Nuñez Garcia D, Meyer R, Richter A, Rudiger A, Schubert M, et al. Screening for delirium with the Intensive Care Delirium Screening Checklist (ICDSC): a re-evaluation of the threshold for delirium. Swiss Med Wkly [Internet]. 2018; 148:w14597. https://www.doi.org/10.4414/smw.2018.14597

23. Gélinas C, Bérubé M, Chevrier A, Pun B, Ely E, Skrobik, et al. Delirium Assessment Tools for Use in Critically Ill Adults: A Psychometric Analysis and Systematic Review. Crit Care Nurse [Internet]. 2018;38(1):38-49. https: //www.doi.org/10.4037/ccn2018633

24. Van Velthuijsen E, Zwakhalen S, Warnier R, Mulder W, Verhey F, Kempen G. Psychometric properties and feasibility of instruments for the detection of delirium in older hospitalized patients: a systematic review. Int J Geriatr Psychiatry [Internet]. 2016;31(9):974-989. https://www.doi.org/10.1002/gps.4441

25. Jones R, Cizginer S, Pavlech L, Albuquerque A, Daiello L, Dharmarajan K, et al. Assessment of Instruments for Measurement of Delirium Severity. A systematic review. JAMA Intern Med [Internet]. 2019;179(2):231-239. h ttps://www.doi.org/10.1001/jamainternmed.2018.6975

26. Boettger S, Nuñez DG, Meyer R, Richter A, Schubert M, Meagher D, et al. Brief assessment of delirium subtypes: Psychometric evaluation of the Delirium Motor Subtype Scale (DMSS) -4 in the intensive care setting. Palliat Support Care [Internet]. 2017;5:535-543. https://www.doi.org/10.1017/S147895151600105X 
27. Khan B, Perkins A, Gao S, Hui S, Campbell N, Farber M, et al. The Confusion Assessment Method for the ICU-7 Delirium Severity Scale: A Novel Delirium Severity Instrument for Use in the ICU. Crit Care Med [Internet]. 2017;45(5):851-857. https://www.doi.org/10.1097/CCM.0000000000002368

28. Rowley-Conwy G. Barriers to delirium assessment in the intensive care unit: A literature review. Intensive Crit Care Nurs [Internet]. 2018;44(feb.):99-104. doi:http://doi.org/10.1016/j.iccn.2017.09.001

29. Faught D. Delirium: The Nurse's Role in Prevention, Diagnosis, and Treatment. Medsurg Nurs. 2014;23(5):301-305.

30. Faría R, Moreno R. Delirium in intensive care: an under-diagnosed reality. Rev Bras Ter Intensiva [Internet]. 2013;25(2):137-147. https://www.doi.org/10.5935/0103-507X.20130025

31. Arumugam S, El-Menyar A, Al-Hassani A, Strandvik G, Asim M, Mekkodithal A, et al. Delirium in the intensive care unit. J Emerg Trauma Shock [Internet]. 2017;10(1):37-46. https://www.doi.org/10.4103/0974-2700.199 520

32. Selim A, Ely E. Delirium the under-recognised syndrome: survey of healthcare professionals' awareness and practice in the intensive care units. J Clin Nurs [Internet]. 2017;26(5-6):813-824. https://www.doi.org/10.1111/jocn. 13517

33. XingJ, Sun Y, Jie Y, Yuan Z, Liu W. Perceptions, attitudes, and current practices regards delirium in China: A survey of 917 critical care nurses and physicians in China. Medicine (Baltimore) [Internet]. 2017;96(39):1-5. https:// www.doi.org/10.1097/MD.0000000000008028

34. Tostes ICGO, Pereira SRM, Almeida LF, et al. Delirium in intensive care: use of the 'Confusion Assessment Method for the Intensive Care Unit' by the nurse. Rev Fund Care Online [Internet]. 2018;10(1):2-8. https://www.doi .org/10.9789/2175-5361.2018.v10i1.2-8

35. Devlin JW, Fong JJ, Howard EP, Skrobik Y, McCoy N, Yasuda C, et al. Assessment of delirium in the intensive care unit: nursing practices and perceptions. Am J Crit Care [Internet]. 2008;17(6):555-565.

36. Flagg B, Cox L, Mcdowell S, Mwose J, Buelow J. Nursing Identification of Delirium. Clin Nurse Spec [Internet]. 2010;24(5):260-266. https://www.doi.org/10.1097/NUR.0b013e3181ee5f95

37. Rowley-Conwy G. Critical care nurses' knowledge and practice of delirium assessment. Br J Nurs [Internet]. 2017;26(7):412-417. https://www.doi.org/10.12968/bjon.2017.26.7.412.

38. Gesin G, Russell BB, Lin AP, Norton HJ, Evans SL, Devlin JW. Impact of a delirium screening tool and multifaceted education on nurses' knowledge of delirium and ability to evaluate it correctly. Am J Crit Care [Internet]. 2012;21(1):1-11. https://www.doi.org/10.4037/ajcc2012605

39. Lieow J, Chen F, Song G, Tang P, Kowitlawakul Y, Mukhopadhyay A. Effectiveness of an advanced practice nurseled delirium education and training programme. Int Nurs Rev [Internet]. 2019;66(4). https://www.doi.org/1 $0.1111 /$ inr. 12519

40. Van Velthuijsen EL, Zwakhalen SMG, Warnier RMJ, Ambergen T, Mulder WJ, Verhey FRJ, et al. Can education improve clinical practice concerning delirium in older hospitalised patients? Results of a pre-test post-test study on an educational intervention for nursing staff. BMC Med Educ [Internet]. 2018;18(59). https://www.doi.o $\mathrm{rg} / 10.1186 / \mathrm{s} 12909-018-1177-3$

41. Norman SL, Taha AA. Delirium Knowledge, Self-Confidence, and Attitude in Pediatric Intensive Care Nurses. J Pediatr Nurs [Internet]. 2019;46:6-11. https://www.doi.org/10.1016/j.pedn.2019.01.013

42. Ramoo V, Abu H, Rai V, Surat Singh SK, Baharudin AA, Danaee M, et al. Educational intervention on delirium assessment using confusion assessment method-ICU (CAM-ICU) in a general intensive care unit. J Clin Nurs [Internet]. 2018;27(21-22):4028-4039. https://www.doi.org/10.1111/jocn.14525

43. Mansouri P, Javadpour S, Zand F, Ghodsbin F, Sabetian G, Masjedi M, et al. Implementation of a protocol for integrated management of pain, agitation, and delirium can improve clinical outcomes in the intensive care unit: A randomized clinical trial. J Crit Care [Internet]. 2013;28(6):918-922. https://www.doi.org/10.1016/j.jcrc.2 013.06.019 
44. Mistarz R, Eliott S, Whitfield A, Ernest D. Bedside nurse-patient interactions do not reliably detect delirium: an observational study. Aust Crit Care [Internet]. 2011;24(2):126-132. https://www.doi.org/10.1016/j.aucc.201 1.01 .002

45. Spronk PE, Riekerk B, Hofhuis J, Rommes JH. Occurrence of delirium is severely underestimated in the ICU during daily care. Intensive Care Med [Internet]. 2009;35(7):1276-1280. https://www.doi.org/10.1007/s0013 4-009-1466-8.

46. Devlin J, Brummel N, Al-Qadheeb N. Optimising the recognition of delirium in the intensive care unit. Best Pract Res Clin Anaesthesiol [Internet]. 2012;26(3):385-393. https://www.doi.org/10.1016/j.bpa.2012.08.002

47. Grover S, Natasha K. Assessment scales for delirium: A review. World J Psychiatry [Internet]. 2012;2(4):58-70. h ttps://www.doi.org/10.5498/wjp.v2.i4.58

48. Andrews L, Silva SG, Kaplan S, Zimbro K. Delirium monitoring and patient outcomes in a general intensive care unit. Am J Crit Care [Internet]. 2015;24(1):48-56. https://www.doi.org/10.4037/ajcc2015740

49. Elliot SR. ICU delirium: a survey into nursing and medical staff knowledge of current practices and perceived barriers towards ICU delirium in the intensive care unit. Intensive Crit Care Nurs [Internet]. 2014.;30(6):333-338. https://www.doi.org/10.1016/j.iccn.2014.06.004

50. Powell T, Nolan M, Yang G, Tam M, Metter D, Gibran N, et al. Nursing Understanding and Perceptions of Delirium: Assessing Current Knowledge, Attitudes, and Beliefs in a Burn ICU. J Burn Care Res [Internet]. 2019;40(4):471-477. https://www.doi.org/10.1093/jbcr/irz040

51. American Association of Critical-Care Nurses. Assessment and Management of Delirium Across the Life Span. Crit Care Nurse [Internet]. 2016;36(5):14-19. https://www.doi.org/10.4037/ccn2016242

52. Morandi A, Brummel N, Ely E. Sedation, delirium and mechanical ventilation: the 'ABCDE' approach. Curr Opin in Crit Care [Internet]. 2011;17(1):43-49. https://www.doi.org/10.1097/MCC.0b013e3283427243

53. Devlin J, Skrobik Y, Gélinas C, Needham D, Slooter A, Pandharipande P, et al. Clinical Practice Guidelines for the Prevention and Management of Pain, Agitation/Sedation, Delirium, Immobility, and Sleep Disruption in Adult Patients in the ICU. Crit Care Med [Internet]. 2018;46(9):825-873. https://www.doi.org/10.1097/CC M.0000000000003299

54. Martínez F, Donoso A, Márquez C, Labarca E. Implementing a Multicomponent Intervention to Prevent Delirium Among Critically Ill Patients. Crit Care Nurse [Internet]. 2017;37(6):36-46. https://www.doi.org/10.4037/cc n2017531

\section{Notas}

* Artículo de revisión

\section{Licencia Creative Commons CC BY 4.0}

Cómo citar este artículo: Henao-Castaño ÁM, Baquero-Lizarazo AC, González-Pabón N, Burgos-Tulcán GN, Lozano L. Enfermería en la monitorización del delirium en cuidado intensivo adulto: una revisión de alcance. Investig Enferm Imagen Desarr. 2020;22. https://doi.org/10.11144/Javeriana.ie22.emdc 